\title{
High-speed Real-time Spectrum Analysis System Based on FPGA and GPU Parallel Arithmetic
}

\author{
Chen Jingye ${ }^{1, ~ a ~}$, Li Ziyu $^{1, b}$, Chen Lei ${ }^{1, c}$ and Xu Junying ${ }^{1, d}$ \\ ${ }^{1}$ Beijing Aerospace Control Center, Beijing 102206, China;

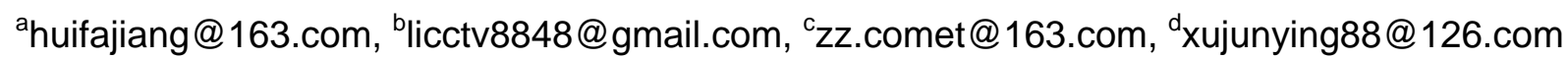

Keywords: real-time spectrum analyzer, FPGA; GPU, parallel computing.

\begin{abstract}
In this paper, a high-speed real-time spectrum analysis system based on GPU parallel computing was developed for the real-time requirements in the application environment of satellite communication spectrum monitoring. The system adopts high-speed ADC and high-performance FPGA as real-time signal acquainting and processing platform and uses high-performance server and GPU as signal analyzing and identifying platform. By using advanced signal processing and recognition algorithms, automatic monitoring and real-time alarms of the spectrum were realized, and this improved communication quality.
\end{abstract}

\section{Introduction}

As communications, aerospace and other fields keep developing, the amount of signals need to be processed in those fields is getting bigger and bigger as well as the calculation of those signals. How to improve the operation speed and operation accuracy of signal processing in those fields has now become a very important research direction. In recent years, it has been very common to use FPGA+GPU for scientific calculation. As GPU keeps developing, GPU has been used in more and more fields. Its powerful parallel computing power can significantly decrease system costs. Due to the special construction of GPU, It can accelerate the processing speed of signals and greatly improve repeated and complex calculation fields. The system uses the powerful parallelism and high-speed data transmission capacity of FPGA. Combining the high-speed parallel data processing capacity, it is possible to satisfy the real-time requirements of current spectrum monitoring.

\section{System Design}

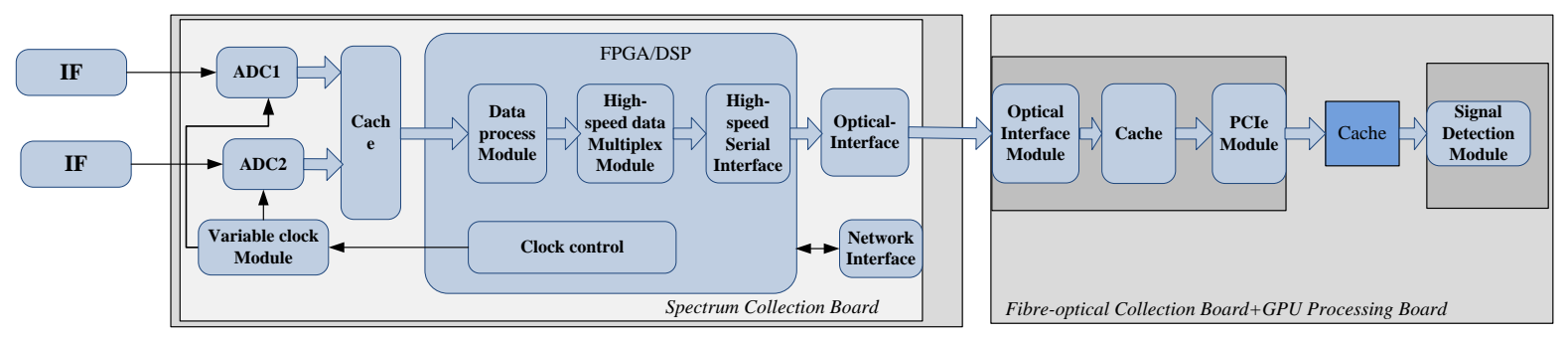

Fig.1 Block diagram of system composition

System Composition. The system is composed of intermediate frequency signal acquisition board, optical fiber interface board and GPU high-speed processing board.

1. Real-time spectrum acquisition board is configured with 2 pieces of high-speed ADC acquisition chips, 2 pieces of FPGA and 1 piece of switching chip. The board supports 2-channel intermediate frequency data acquisition and transmission and 2-way optical fiber transmission. Intermediate frequency signals will be transferred to digital signals based on certain sampling strategy and sent to GPU processing board.

2. Processing equipment includes 1 piece of optical interface board and 1 piece of GPU high-speed processing board, which have the functions of receiving original signals and analyzing and 
comparing modulation domain and frequency domain. Original signals are stored based on sampling time, and frequency domain signal of abnormal storage will be checked afterwards ${ }^{[1][2][3]}$.

Real-time Spectrum Acquisition Board. Real-time spectrum acquisition board is mainly used for the high-speed acquisition of intermediate frequency signals in channel 2, and then original signals will be transmitted through the optical interface to process server. A board card is composed two parts: high-speed ADC acquisition daughter board and FPGA signal processing transmission carrier board. The two parts are connected through FMC connector ${ }^{[5]}$.

Real-time spectrum acquisition board is 6U VPX standard 2-channel high-speed data acquisition board which can be used for 2-channel signal acquisition, storage, processing and transmission. The block diagram of real-time spectrum acquisition board is shown in Figure 2:

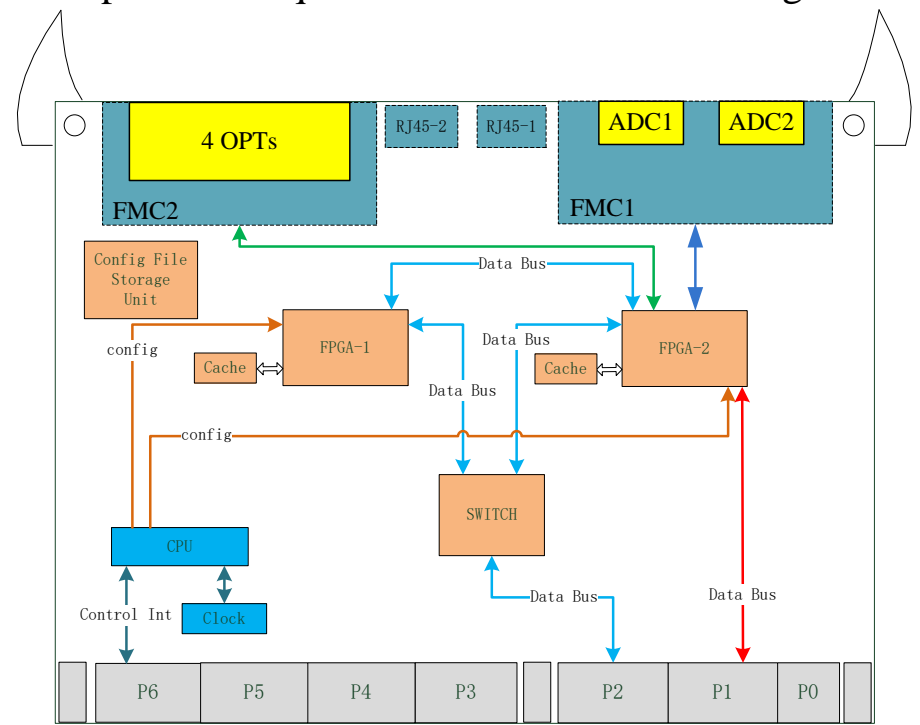

Fig.2 Block diagram of real-time spectrum acquisition board

Optical fiber interface board. Optical interface board receives the spectrum data transmitted from spectrum acquisition devices through optical ports, and the data is transmitted to the machine through PCIe data bus. The board supports 4-way optical fiber channel, and maximum 5Gbps full-duplex data transmission is realized. The block diagram of board card is shown in Figure 3:

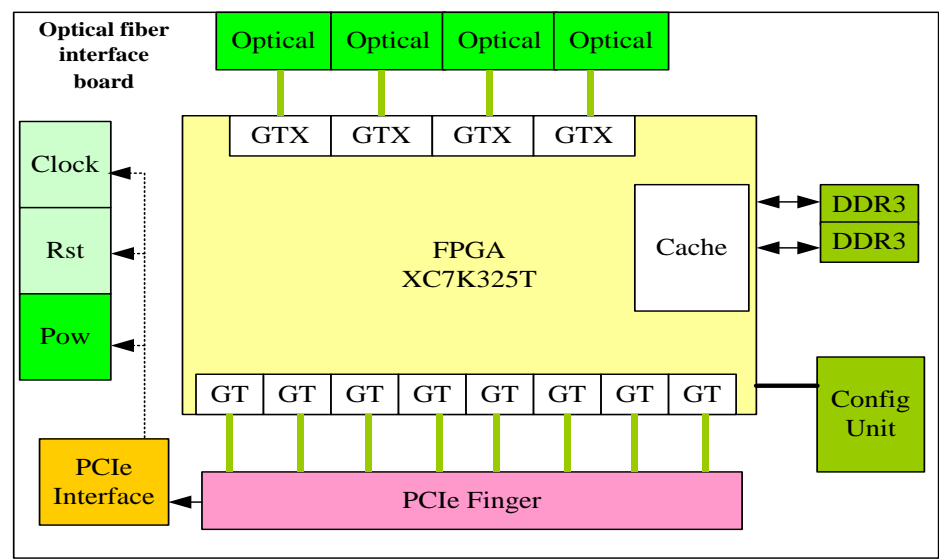

Fig.3 Block diagram of optical fiber PCIe receiver board

Xilinx Company integrated Endpoint Block Plus for PCI-Express core on Kintex-7 Series chip, realizing the interface between users and PCIe data bus. Using this module, the customer can ignore the physical layer and data link layer of PCIe data bus. And the most important BMD module design can be realized through the transaction layer interface provided by the module ${ }^{[4]}$.

GPU high-speed processing board. Based on the instantaneity and processing requirements of the system and the design principle of scalability, we choose GPU high-speed processing board card for the processing of acquired backend time domain and frequency domain signals, extraction of characteristics parameters and comparison of alarms. 
Table 1 is the comparison of processing performance of FFT conversion using GPU and DSP chips.

Table 1 FFT conversion performance comparison of GPU and DSP

\begin{tabular}{ccc}
\hline $\begin{array}{c}\text { FFT operation } \\
\text { points }\end{array}$ & $\begin{array}{c}\text { GPU GTX690 } \\
\text { processing time (ms) }\end{array}$ & $\begin{array}{c}\text { DSP TMS320c6678 } \\
\text { processing time (ms) }\end{array}$ \\
\hline $256 \mathrm{~K}$ & 0.107 & 1.1026 \\
$128 \mathrm{~K}$ & 0.064 & 0.6389 \\
$64 \mathrm{~K}$ & 0.034 & 0.3346 \\
$32 \mathrm{~K}$ & 0.0268 & 0.2665 \\
\hline
\end{tabular}

From the above table it can be seen that the use of GPU for FFT conversion has outstanding advantages. Compared with traditional FPGA+DSP, it has higher processing speed, and in GPU development environment CUDA completely support VS2010. On the other hand, using GPU for processing is scalable. By simply adding the front-end acquisition devices, it is possible to realize multi-channel data processing. The system has relatively high requirements for instantaneity and processing functions.

\section{Experimental Results and System Performance Test}

Modulation recognition and detection performance.1) Modulation model: BPSK, QPSK, OQPSK, 8PSK, 16QAM, 16APSK.

2) Minimum detection level: 5dB Eb/No(QPSK),1dB Eb/NO(BPSK,OQPSK, 8PSK, 16QAM, 16APSK).

Calculation Accuracy.1) Carrier frequency estimates: It is required for the system that all modulation signals shall use decision-feedback phase locked loop for estimation. The estimated deviation of emulation proof is within $\pm 300 \mathrm{~Hz}$, ad the relative estimation precision is $\pm 4.3 \times 10-6$.

2) Signal to noise ratio estimates: It is required for the system that all modulation signals shall carry out estimation based on singular value decomposition algorithm, and the estimated deviation of emulation proof is within $\pm 0.5 \mathrm{~dB}$.

3) Symbol rate estimation: It is required for the system that all modulation signals shall carry out estimation based on cyclic spectrum algorithm, and the relative estimated accuracy of emulation proof is $\pm 2.4 \times 10-4$.

4) Bandwidth estimates: It is required for the system that all modulation signals shall carry out estimation based on conventional centric algorithm, and the relative estimated accuracy of emulation proof is $\pm 0.5 \% \mathrm{OBW}$.

5) Recognition probability: It is required for the system that all modulation signals shall carry out estimation based on higher-order cumulate algorithms, zero defect recognition can be carried out when Eb/N0 of emulation proof is not less than $5 \mathrm{~dB}$.

Spectrum measurement display. It is possible to automatically display the bandwidth, center frequency, power, carrier to noise ratio, Eb / No, modulation method and other key characteristic parameters of the carrier wave, wherein the lower part of the screen displays the total power measured value in current monitoring channel. Meanwhile, it has the mark point function of spectrum analyzer. Spectrum display screen can display the retention curve of the spectrum's maximum value and minimum value in current monitoring channel, and this is to the benefit of timely observation when an error occurs. Spectrum measuring function is shown in Figure 4 


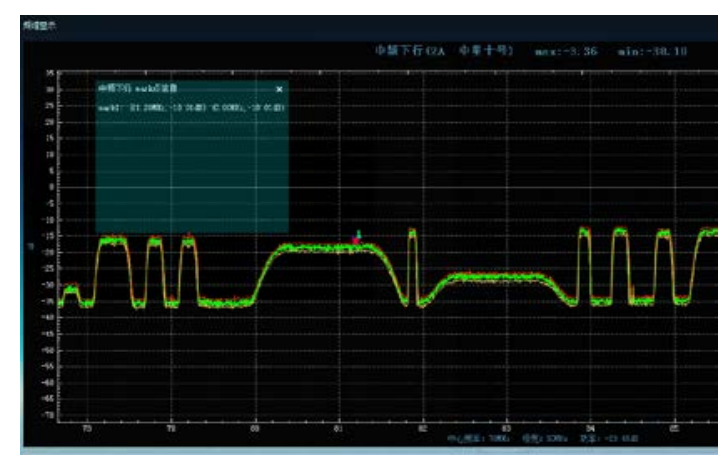

Fig.4 Spectrum measuring function

Display of carrier characteristic parameters. Spectrum monitoring system is able to identify characteristic parameters such as modulation mode and code rate based on priori knowledge. Identifiable modulation modes include: BPSK, QPSK, 8PSK, 16QAM and 16APSK. At the same time, the characteristic parameters of RF Power, bandwidth, frequency and C/No can also be displayed.

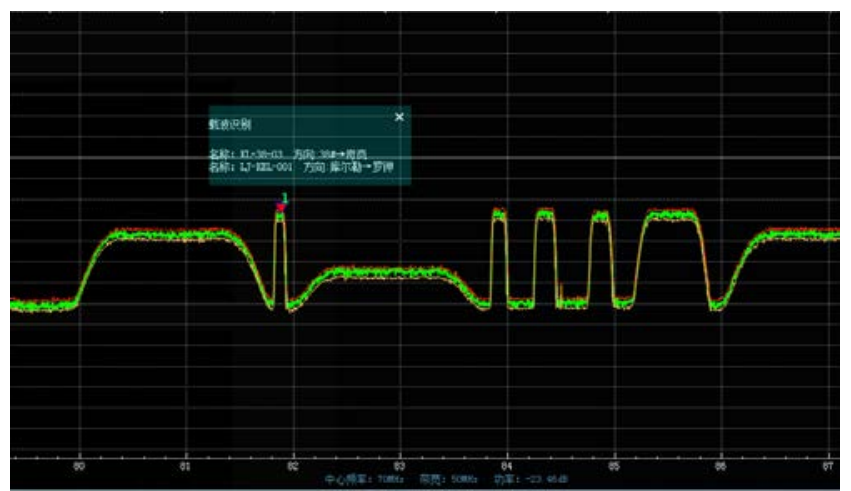

Fig.5 Identification interface of the features of carrier waves

\section{Conclusion}

Concerning the requirements for bandwidth and high instantaneity of high-speed real-time spectrum analyzing system, the paper put forward a high-speed parallel signal processing hardware system based on large-scale FPGA and high-performance GPU. By using the high-speed optical fiber interface based on EBP technology, the data transmission between spectrum acquisition board and GPU processing board was completed, realizing high-resolution and high-accuracy FFT conversion for bandwidth intermediate frequency signals. Meanwhile, the characteristic parameters of carrier waves can be extracted under certain conditions.

\section{References}

[1] Bing Li, WeiWang, Zengping Chen. Design and Implement of BIT for Radar Data Acquisition and Feature Extraction System.Electronic Engineer，30(1),2004

[2] XianFu Song, TianShuang Qiu, Hong Tang, Ying Guo. Study on the Automatic Recognition of the Wireless Radio Signal Modulation Method . China Science Paper Online, 2001

[3] Rui Zhao, WeiNing Lu. Study on the Automatic Recognition of Common Satellite Signal Digital Modulation Method. Aerospace Electronic Warfare, 24(6), 2008:12-20

[4] Xilinx. Kintex-7 FPGAs Data Sheets

[5] Ti. ADS5463 Data Sheets 